\title{
Formación STEM en el grado de maestro: una experiencia docente
}

\author{
Pre-service teacher training in STEM: a teaching experience
}

\author{
Irene Ferrando Palomares \\ Universitat de València \\ irene.ferrando@uv.es

\section{Desamparados Hurtado Soler} \\ Universitat de València \\ amparo.hurtado@uv.es \\ María José Beltrán Meneu \\ Universitat Jaume I \\ mmeneu@uji.es
}

Fecha presentación: 12/11/2017 | Aceptación: 16/02/2018 |Publicación: 22/06/2018

\section{Resumen}

El objetivo de este trabajo es evaluar el diseño de la asignatura TIC como recurso didáctico en ciencias y matemáticas impartida en el grado en Maestro/a en Educación Primaria de la Universitat de València, durante los cursos 2013-14 y 2014-15, a partir del análisis de los trabajos del alumnado y de sus respuestas a un cuestionario. La finalidad de la asignatura es formar a maestros del ámbito STEM capaces de abordar una enseñanza integrada de las ciencias y las matemáticas mediante las Tecnologías de la Información y la Comunicación (TIC). Los resultados muestran un elevado interés del alumnado por el enfoque de la materia, el diseño de actividades y el trabajo cooperativo. No obstante, se detectan algunas dificultades en los futuros maestros a la hora de trabajar de forma interdisciplinar algunos contenidos de ciencias y de matemáticas en determinados cursos de primaria. La introducción de las TIC en la enseñanza de contenidos STEM es una oportunidad para desarrollar la competencia digital.

Palabras clave: Formación inicial; Educación Primaria; Propuestas didácticas; STEM; TIC

\section{Resum}

L'objectiu d'aquest treball és avaluar el disseny de l'assignatura TIC com a recurs didàctic en ciències i matemàtiques impartida en el grau en Mestre/a en Educació Primària de la Universitat de València, durant els cursos 2013-14 i 2014-15, a partir de l'anàlisi dels treballs de l'alumnat i les respostes a un qüestionari. La finalitat de l'assignatura és formar als mestres en l'àmbit STEM abordant l'ensenyament integrat de les ciències i les matemàtiques fent ús de les Tecnologies de la Informació i la Comunicació (TIC). Els resultats mostren un elevat interès de l'alumnat per la matèria, el disseny d'activitats i el treball cooperatiu. No obstant això, es detecten algunes dificultats a l'hora de treballar de forma interdisciplinària alguns continguts de ciències i de matemàtiques en determinats cursos de primària. L'ús de les TIC en l'ensenyament de continguts STEM és una oportunitat per desenvolupar la competència digital.

Paraules clau: Formació inicial; Educació Primària; Propostes didàctiques; STEM; TIC

\section{Abstract}

The aim of this work is to evaluate the design of the subject ICT as a teaching resource in science and mathematics taught in the Bachelor's Degree in Primary Education of the Universitat de València, during the academic courses 201314 and 2014-15. The analysis is based on the works of the students and their responses to a questionnaire. The purpose of the subject is to train pre-service teachers in the STEM discipline to make them able to teach Science and Mathematics in an integrated way throughout the Information and Communication Technologies (ICT). The results show

${ }^{1}$ Este trabajo ha sido financiado por el proyecto GVA, grupo de investigación emergente GV/2016/129 y el proyecto de innovación educativa de la Universitat de València L'Hort 2.0 (UV-SFPIE_RMD16-418948). 
Beltrán Meneu, María José; Hurtado Soler, Desamparados y Ferrando Palomares, Irene (2018) “Formación STEM en el grado de maestro: una experiencia docente". @tic revista d'innovació educativa, 20, 35-42.

a high interest of the students in the subject, the design of activities and the cooperative work. However, some difficulties are identified related to the interdisciplinary teaching of science and mathematics in elementary courses. The application of ICT in the STEM teaching is an opportunity to develop the teaching competence.

Key Words: Pre-service teacher training; Elementary Education; Teaching proposals; STEM; ICT

\section{Introducción}

La implantación del Plan Bolonia ha supuesto un reto para las universidades españolas, que han tenido que armonizar su sistema educativo a las exigencias metodológicas de la Unión Europea. Este modelo se centra en el alumno como protagonista del proceso de enseñanza-aprendizaje de forma que asuma un papel más activo y crítico frente al modelo tradicional pasivo y receptivo. En este contexto, los estudios de magisterio se han ampliado, pasando de tres a cuatro años, como el resto de grados. Ello ha incrementado la carga docente de algunas áreas de conocimiento. En concreto, los alumnos del grado en Maestro/a en Educación Primaria de la Universitat de València (UV) pueden elegir varios itinerarios formativos en tercero y cuarto, y dos de ellos (el de especialista en Ciencias y Matemáticas y el de TIC) incluyen la asignatura "TIC como recurso didáctico en ciencias y matemáticas" en último curso.

La introducción de esta asignatura está relacionada con el desarrollo de la integración de diferentes disciplinas del ámbito científico-tecnológico en una sola denominada STEM por su acrónimo en inglés de Ciencia, Tecnología, Ingeniería y Matemáticas. En efecto, en los últimos años se insiste, desde diferentes organismos ( $p$. ej. informe Eurydice, 2012), en la importancia de promover el aprendizaje integrado de las matemáticas y las ciencias eliminando las barreras que separan las disciplinas. Tsupros et al. (2009) definen la educación en STEM como una aproximación interdisciplinar al aprendizaje en el que los conocimientos académicos rigurosos se combinen con lecciones cercanas al mundo real de forma que los estudiantes apliquen la ciencia y las matemáticas en contexto. Sin embargo, aunque se han realizado estudios enfocados en el aprendizaje STEM desde el punto de vista del alumnado, todavía hay pocos trabajos relativos a la formación del maestro STEM (Brown, 2012).

Así, el trabajo que presentamos pretende abordar este aspecto, describiendo y evaluando el desarrollo de una asignatura cuyo objetivo es que los futuros maestros aprendan a diseñar propuestas didácticas a través de las cuales los niños de educación primaria adquieran, de forma integrada y a través de las TIC, contenidos de matemáticas y de ciencias.

Desde nuestro punto de vista, sólo a través de cambios en la formación de los maestros se obtendrán resultados nuevos en las aulas. Además "las materias de carácter científico no sólo deben ocuparse del conocimiento e interacción con el mundo físico" (De Pro, 2012), por ello, consideramos fundamental enseñarles a adquirir conocimientos sobre el uso de las TIC como herramienta fundamental de la actividad docente al tiempo que a desarrollar propuestas didácticas que conjuguen contenidos de ciencias y matemáticas.

\section{Antecedentes y referentes teóricos}

Pese a la importancia que se ha dado en los últimos años a la enseñanza integrada de disciplinas científicas, tal y como muestran algunos trabajos (Brown, 2012), la mayoría de las investigaciones se han centrado en el aprendizaje STEM en estudiantes de educación secundaria, con escasa atención a los maestros. Esto contrasta significativamente con la importancia, ratificada en trabajos como el de Reeve (2015), de que los maestros reciban una correcta formación en este ámbito. Tal y como destacan Rinke et al. (2016), existe poca investigación sobre cómo preparar de manera efectiva a los futuros docentes para convertirse en maestros STEM. Estos autores, al igual que Niess (2005) describen en su trabajo una propuesta de intervención con futuros maestros similar a la que proponemos en este artículo, pero en un contexto diferente teniendo en cuenta las particularidades de los sistemas educativos de cada país.

Tal y como se explica en Moomaw (2013, p. 4), la enseñanza STEM implica que los maestros planeen cuidadosamente las experiencias de enseñanza y aprendizaje a partir de los contenidos de ciencias y matemáticas que quieren introducir, utilizando la tecnología como una herramienta más del proceso de aprendizaje. Por tanto, el diseño de secuencias didácticas integradas debe centrarse en materiales, situaciones y experiencias que sean interesantes y que promuevan el aprendizaje significativo.

La modelización matemática entendida como el proceso de matematización, interpretación, validación y generalización de situaciones reales o sistemas complejos (Lingefjärd, 2006) promueve desarrollos conceptuales significativos en los estudiantes. Es por ello que, recientemente, muchos investigadores han incidido en la importancia de implementar tareas de modelización para desarrollar el pensamiento analítico (Lesh y Zawojewski, 2007). Estos mismos argumentos son utilizados para enfatizar la importancia de una enseñanza integrada de las disciplinas que componen el ámbito STEM (Capraro y Slough, 2008, English y Mousoulides, 2011). En efecto, las tareas de modelización, involucran procesos relacionados con las disciplinas STEM y, por tanto, resultan idóneas en las propuestas que pretenden promover un aprendizaje integrado (Kertil y Gurel, 2016).

Siguiendo esta idea, pretendemos que los futuros maestros diseñen secuencias de tareas que se construyan a partir de una situación real en la que se plantee un problema de aprendizaje y se realice un tratamiento conceptual. Hay que tener en cuenta que tanto las matemáticas como las ciencias experimentales requieren de un tratamiento lógico, secuencial y ordenado, por ello las secuencias deben organizarse adecuadamente buscando conectores con temas de la realidad para permitir la construcción de significados (Moomaw, 2013 y Díaz Barriga, 2011).

En cuanto a la estructura de la secuencia hay que tener en cuenta la integración de dos elementos paralelos (Couso, 2013 y Ros, 2013): la secuencia de actividades para el aprendizaje y la evaluación del mismo. De esta forma se pueden detectar dificultades que permitirán reorganizar el avance de la secuencia. Los trabajos realizados por los estudiantes constituyen elementos de 
Beltrán Meneu, María José; Hurtado Soler, Desamparados y Ferrando Palomares, Irene (2018) “Formación STEM en el grado de maestro: una experiencia docente". @tic revista d'innovació educativa, 20, 35-42.

evaluación de modo que la secuencia integra principios de aprendizaje con las tres dimensiones de la evaluación: diagnóstica, formativa y sumativa.

\section{Objetivos y estructura de la asignatura}

La asignatura, coordinada por docentes de dos departamentos diferentes (Didáctica de las Ciencias y Didáctica de las Matemáticas), tiene una duración de 14 semanas lectivas con dos sesiones de dos horas semanales. Las 6 primeras sesiones están enfocadas a introducir, mediante clases teórico-prácticas, los fundamentos sobre el diseño y la evaluación de propuestas didácticas multidisciplinares así como algunos conceptos prácticos relativos al uso de las TIC. Puesto que se trata de conseguir que los futuros maestros aprendan a diseñar una propuesta didáctica contextualizada para enseñar de forma integrada contenidos de ciencias y de matemáticas a través de las TIC, el desarrollo de la asignatura está centrado en la práctica. A través de las actividades que se realizan, se espera que los futuros maestros:

1. Integren y utilicen los conocimientos adquiridos, al seleccionar y diseñar propuestas didácticas que favorezcan aprendizajes con sentido para alumnos de cualquier curso de Educación Primaria.

2. Propongan secuencias de actividades en las que se trabajen de forma integrada contenidos de ciencias y matemáticas adecuados al curso al que se dirige la propuesta.

3. Analicen con sentido crítico las propuestas didácticas que elaboren y sean capaces de plantear una autoevaluación de la misma.

4. Sean capaces de usar las herramientas TIC adecuadas para llevar a cabo la secuencia de actividades.

5. Avancen en el desarrollo de las habilidades para comunicar de forma efectiva el resultado final de su propuesta didáctica.

Para conseguir estos objetivos, se plantea el diseño de una propuesta didáctica contextualizada en un entorno o tema real y cotidiano que será el marco o eje conductor de las secuencias de aprendizaje.

Durante las sesiones centradas en los fundamentos de diseño de las propuestas didácticas planteamos a los estudiantes un esquema general que les servirá para vertebrar las actividades de su propuesta. Este esquema, descrito en Jorba y Sanmartí (1996) está basado en la utilización de cuatro tipos de actividades que se organizan secuencialmente para, finalmente, trabajar de forma completa los contenidos didácticos establecidos. Así, una secuencia de actividades debe contener actividades de exploración, de introducción de conceptos, de estructuración y de aplicación.

Las actividades de exploración son aquellas que permiten que el alumno se familiarice con los contenidos que va a aprender a través del análisis de situaciones concretas cercanas a sus intereses. Estas actividades, fundamentales en el proceso de aprendizaje, permiten que el profesor identifique cuáles son los puntos fuertes y débiles de sus estudiantes respecto a los contenidos que va a desarrollar (Driver, 1988).

Las actividades de introducción de conceptos permiten mostrar a los estudiantes aspectos relacionados con nuevos conceptos y procedimientos, así como su relación con contenidos conocidos.

Las actividades de estructuración ayudan a asimilar los contenidos introducidos relacionándolos entre sí, fundamentalmente mediante esquemas o problemas.

Por último, las actividades de aplicación consisten en utilizar los contenidos trabajados en otros contextos o situaciones. Es muy importante que estas actividades permitan a los alumnos comparar sus conocimientos iniciales con los adquiridos. Las tareas de modelización resultan idóneas para aplicar contenidos STEM.

Durante estas sesiones teóricas, se trabaja también la evaluación, centrándose en la reflexión sobre la utilidad de la retroalimentación como mecanismo de evaluación. Este apartado se inicia con la lectura de investigaciones y textos enfocados en la influencia que tiene la forma de evaluar sobre la forma de estudiar. A partir de esta reflexión, los estudiantes analizan ejercicios de las pruebas liberadas de PISA y TIMSS en ciencias y matemáticas identificando las dificultades que encuentran los alumnos de primaria para afrontarlas. Esta reflexión sirve de base para identificar las fases de evaluación, sus características y las herramientas disponibles con el fin de planificar de forma adecuada el proceso de evaluación en la propuesta didáctica que van a elaborar.

Finalmente, se aborda el manejo de los programas que pueden utilizar en sus propuestas. Debido a su gran abundancia, nos centramos en la revisión de los más utilizados en el ámbito educativo, promoviendo que los estudiantes seleccionen y utilicen programas accesibles, con interfaz amigable y navegable y de licencia libre.

Conviene destacar que el reparto de las 6 sesiones teórico-prácticas es diferente en función del itinerario que realizan. En efecto, los alumnos del itinerario de TIC ya han cursado materias relacionadas con el manejo de entornos digitales mientras que los alumnos del itinerario de Ciencias y Matemáticas han superado asignaturas relacionadas con los fundamentos de diseño de las propuestas didácticas multidisciplinares. Para homogeneizar los conocimientos de los alumnos con el fin de que puedan abordar con garantías el desarrollo de la propuesta didáctica, las sesiones dirigidas al grupo que cursa el itinerario TIC se centrarán en la fundamentación del diseño de la propuesta didáctica y la evaluación, mientras que las dirigidas al grupo que cursa el itinerario de ciencias y matemáticas se centrarán en enseñarles el manejo de los programas.

Una vez los estudiantes tienen una idea clara de cómo enfocar su propuesta se dedican a trabajar de forma autónoma en grupos de tres o cuatro alumnos. En primer lugar, escogen un contexto sobre el cual diseñar una serie de actividades a través de las cuales se trabajan de forma integrada, a través de las TIC, contenidos de matemáticas y de ciencias. Los contextos escogidos por los estudiantes son diversos y responden a sus intereses. El hecho de trabajar sobre un contexto real y cercano les permite enfocar de forma más clara el diseño de actividades siguiendo la secuencia explicada previamente. A continuación, cada grupo debe escoger el formato digital sobre el cual van a realizar su propuesta. La idea es que el diseño, la accesibilidad y navegabilidad del soporte sea motivador y atractivo centrándose en la tipología y nivel del alumnado al que va dirigido.

Para consolidar el aprendizaje en lo que al diseño de propuestas multidisciplinares se refiere, se pide a los alumnos que se enfrenten a tres retos: incluir en su 

grado de maestro: una experiencia docente". @tic revista d'innovació educativa, 20, 35-42.

propuesta un contenido curricular de ciencias y otro de matemáticas fijados de antemano por las docentes (ellos deben completar su propuesta incluyendo, al menos, un contenido más de cada disciplina), diseñar una actividad de modelización y plantear una actividad que pondrán en práctica con sus compañeros en el huerto escolar. Esta sesión es interesante ya que es fundamental que los futuros maestros se pongan, a modo de un juego de rol, en el lugar de sus futuros alumnos. No es sino enfrentándose a las tareas que van a diseñar cuando van a observar el interés real de éstas. En este juego los alumnos toman tres roles que se van alternando: por una parte, son los maestros, mostrando a sus compañeros el desarrollo de la actividad, por otro son los alumnos receptores y actores de la misma, y finalmente son los evaluadores, usando una rúbrica a través de la cual valoran la idoneidad y el desarrollo de la actividad.

\section{Evaluación}

La asignatura se evalúa poniendo un énfasis especial en el desarrollo de la misma, se trata de una evaluación continuada en la que se establece una retroalimentación constante entre alumnos y docentes. Desde el inicio del curso se muestra la rúbrica de evaluación que abarca diferentes aspectos detallados en la Figura 1.

\begin{tabular}{|c|c|c|c|}
\hline Sesiones & Herramienta de evaluación & Criterios / aspectos evaluados & $\begin{array}{l}\text { \%nota } \\
\text { final }\end{array}$ \\
\hline \multirow{2}{*}{$\begin{array}{l}1 \text { a } 6 \\
\text { Clases } \\
\text { teórico- } \\
\text { prácticas }\end{array}$} & $\begin{array}{l}\text { - Examen de desarrollo } \\
\text { escrito }\end{array}$ & $\begin{array}{l}\text { - Comprensión de los fundamentos } \\
\text { teóricos } \\
\text {-Comunicación verbal }\end{array}$ & 5 \\
\hline & $\begin{array}{l}\text { - Actividades de resolución } \\
\text { de problemas de } \\
\text { modelización } \\
\text { - Debates y reflexión crítica }\end{array}$ & $\begin{array}{l}\text { - Argumentación oral } \\
\text { - Aplicación de contenidos }\end{array}$ & 5 \\
\hline $\begin{array}{l}7 \text { a } 25 \\
\text { Desarrollo } \\
\text { de la } \\
\text { propuesta }\end{array}$ & $\begin{array}{l}\text { - Revisión continuada del } \\
\text { desarrollo del trabajo en } \\
\text { el aula y en tutorías } \\
\text { (individuales y grupales) } \\
\text { - Observación directa } \\
\text { - Proyecto final mediante } \\
\text { rúbrica de evaluación }\end{array}$ & $\begin{array}{l}\text { - Justificación del contexto } \\
\text { - Selección y relación de los contenidos } \\
\text { - Estructura de la secuencia (tipos y } \\
\text { orden de actividades) } \\
\text { - Adecuación al nivel educativo } \\
\text { - Accesibilidad y navegabilidad de la } \\
\text { interfaz } \\
\text { - Diversidad de recursos TIC } \\
\text { - Procedimiento de evaluación }\end{array}$ & 60 \\
\hline $\begin{array}{l}\text { Juego de } \\
\text { rol (huerto } \\
\text { escolar) }\end{array}$ & $\begin{array}{l}\text { - Cuestionario de } \\
\text { evaluación } \\
\text { - Coevaluación } \\
\text { - Observación directa }\end{array}$ & $\begin{array}{l}\text { - Creatividad y motivación } \\
\text { - Objetivos y contenidos } \\
\text { - Viabilidad y utilidad } \\
\text { - Recursos utilizados } \\
\text { - Organización temporal }\end{array}$ & 10 \\
\hline $\begin{array}{l}26 \text { a } 28 \\
\text { Exposicion } \\
\text { es orales }\end{array}$ & $\begin{array}{l}\text { - Rúbrica de evaluación } \\
\text { - Grabación audiovisual }\end{array}$ & $\begin{array}{l}\text { - Creatividad y escenificación } \\
\text { - Comunicación oral } \\
\text { - Recursos audiovisuales y tecnológicos }\end{array}$ & 20 \\
\hline
\end{tabular}

Figura 1. Planificación, herramientas y ponderación de la evaluación

\section{Análisis y evaluación de la asignatura}

La evaluación de una asignatura una vez finalizada es fundamental para incorporar mejoras en el curso siguiente. Tal y como señala Treffers (1987), la investigación en el área de las propuestas didácticas incluye las fases de diseño, desarrollo, evaluación y revisión de las secuencias de aprendizaje. Mientras la fase de diseño viene sustentada por un marco teórico, los datos recogidos durante el desarrollo de la asignatura nos sirven como punto de partida para realizar un análisis crítico de la misma. Esta retroalimentación se utiliza para refinar el diseño y poder incorporar cambios en un nuevo curso.

Para la realización de este análisis crítico, nos basaremos en datos recogidos durante los cursos 201314 y 2014-15. Contamos con una muestra de 161 alumnos que cursaron la asignatura, correspondientes a cinco grupos naturales de clase, dos del itinerario de especialización en Ciencias y Matemáticas y tres del de TIC. Por un lado, analizaremos, en base a los 42 trabajos realizados por los estudiantes de ambos cursos, aspectos de las propuestas didácticas diseñadas que pueden dar información acerca de las competencias adquiridas por los alumnos relacionadas con el diseño de una propuesta didáctica STEM y por otro, describiremos los resultados obtenidos en un cuestionario en línea dirigido a evaluar algunos aspectos relativos a la enseñanza integrada de ciencias y matemáticas (este cuestionario fue contestado voluntariamente por estudiantes del curso 2014-15). La incorporación del cuestionario durante el segundo curso fue motivada por la observación de una carencia de información suficiente para evaluar el desarrollo de la asignatura en base, únicamente, a las propuestas diseñadas por los estudiantes.

Diseño y análisis de los resultados del cuestionario en línea

Los alumnos accedieron al cuestionario a través de un formulario en línea creado con la herramienta Google Forms, los detalles de las preguntas pueden consultarse en el documento anexo. El cuestionario consta de 27 ítems con respuesta en una escala Likert 0-5 (desde muy en desacuerdo hasta muy de acuerdo). Pretende evaluar aspectos tanto generales relativos al desarrollo de la asignatura, como específicos acerca del diseño de su propuesta didáctica y del uso de TIC. Para el diseño del cuestionario nos hemos basado en un cuestionario validado en otro estudio (Yasar et al., 2006) que hemos adaptado al contexto de nuestra experiencia.

A continuación, presentamos un análisis descriptivo de los resultados de las respuestas recogidas de los 38 estudiantes que accedieron a contestar el cuestionario basado en la puntuación media obtenida en cada ítem. La Figura 2 recoge la opinión de los alumnos acerca de aspectos generales.

\begin{tabular}{|l|l|}
\hline & Media \\
\hline La asignatura es interesante & 4.38 \\
\hline La forma de evaluación es adecuada & 4.21 \\
\hline Las actividades son interesantes & 3.97 \\
\hline $\begin{array}{l}\text { Las clases están bien organizadas y se han logrado los } \\
\text { objetivos }\end{array}$ & 4.10 \\
\hline Los contenidos son interesantes & 3.74 \\
\hline Se fomenta el trabajo cooperativo & 4.74 \\
\hline
\end{tabular}

Figura 2. Opinión de los alumnos acerca de aspectos generales de la asignatura

Observamos que el grado de satisfacción de los alumnos es elevado, sin embargo, los promedios de las valoraciones obtenidas en los ítems que evalúan el interés de los contenidos y las actividades es menor. Cabe destacar la opinión generalizada de que el enfoque de la asignatura fomenta el trabajo cooperativo.

A continuación, en la Figura 3 , se describen las respuestas a los ítems relacionados con aspectos específicos de la propuesta didáctica diseñada por los estudiantes.

\begin{tabular}{|l|l|}
\hline & Media \\
\hline La estructura de la propuesta es adecuada & 4.5 \\
\hline Me ha resultado difícil elegir el contexto & 2.10 \\
\hline $\begin{array}{l}\text { Es interesante fijar un contenido de ciencias y uno de } \\
\text { matemáticas común a todas las propuestas }\end{array}$ & 3.32 \\
\hline Es difíil relacionar los contenidos fijados con el contexto & 3.32 \\
\hline El tiempo de trabajo es adecuado & 3.55 \\
\hline Es difíil trabajar en grupo & 1.34 \\
\hline Las fechas de exposición y entrega son adecuadas & 4.21 \\
\hline Las exposiciones previas son útiles & 3.29 \\
\hline Los ítems de la hoja de evaluación de la propuesta son adecuados & 3.47 \\
\hline
\end{tabular}

Figura 3. Opinión de los alumnos acerca del diseño y desarrollo de la Propuesta Didáctica 
Beltrán Meneu, María José; Hurtado Soler, Desamparados y Ferrando Palomares, Irene (2018) “Formación STEM en el grado de maestro: una experiencia docente". @tic revista d'innovació educativa, 20, 35-42.

Aunque los alumnos consideran que la estructura de la propuesta mediante secuencias de aprendizaje es adecuada, se detectan dificultades en el diseño y desarrollo de la misma. La primera de ellas surge a la hora de seleccionar el contexto, observamos además que tienen dificultades a la hora de integrar, organizar y desarrollar los contenidos en el mismo.

Finalizamos el análisis con la evaluación de los ítems referidos al uso de las TIC (Figura 4). La mayoría de alumnos consideran que el curso les ha permitido descubrir nuevas herramientas distintas a las utilizadas en cursos anteriores, aunque casi todos han encontrado dificultades técnicas.

\begin{tabular}{|l|l|}
\hline & Media \\
\hline Se han descubierto nuevas herramientas TIC para crear actividades & 4.39 \\
\hline $\begin{array}{l}\text { Se han utilizado herramientas TIC distintas a las usadas en cursos } \\
\text { anteriores }\end{array}$ & 4.37 \\
\hline Se han encontrado dificultades técnicas con el uso de las TIC & 3.26 \\
\hline Se ha logrado superar las dificultades técnicas con el uso de las TIC & 3.95 \\
\hline Se han mejorado las competencia en el uso de las TIC & 4.39 \\
\hline
\end{tabular}

Figura 4. Opinión de los alumnos acerca del uso de las TIC en la asignatura

\section{Análisis de las propuestas didácticas}

En este apartado analizamos algunos aspectos de las 42 propuestas didácticas realizadas por los grupos de estudiantes. Analizamos el nivel educativo al que se dirigen, los contenidos de ciencias y matemáticas, el contexto y los recursos TIC utilizados. Estos aspectos, relacionados con los objetivos 1, 2 y 4 de la asignatura presentados previamente, nos aportarán información acerca de las competencias adquiridas por los alumnos y relacionadas con el diseño de una propuesta didáctica STEM.

Respecto al nivel escogido, hemos observado una tendencia a evitar los primeros cursos de Educación Primaria (Figura 5). Se observa que, en algunas propuestas, no se especifica el curso al que se dirige.

\begin{tabular}{|l|l|l|l|l|l|l|l|}
\hline Curso & $\mathbf{1}^{\circ}$ & $2^{\circ}$ & $\mathbf{3}^{\circ}$ & $4^{\circ}$ & $5^{\circ}$ & $6^{\circ}$ & $\begin{array}{l}\text { No se } \\
\text { indica }\end{array}$ \\
\hline $\mathbf{N}^{\circ}$ grupos & 0 & 4 & 2 & 8 & 6 & 12 & 10 \\
\hline
\end{tabular}

Figura 5. Cursos en los que se han contextualizado las Propuestas Didácticas

Además de los contenidos curriculares fijados por las responsables de la asignatura, los futuros maestros tenían que escoger, al menos, un contenido más de cada área, uno del currículo de ciencias y otro de matemáticas. En la Figura 6 relacionamos, en una tabla de doble entrada, los bloques de matemáticas y de ciencias correspondientes a los contenidos escogidos en las propuestas analizadas.

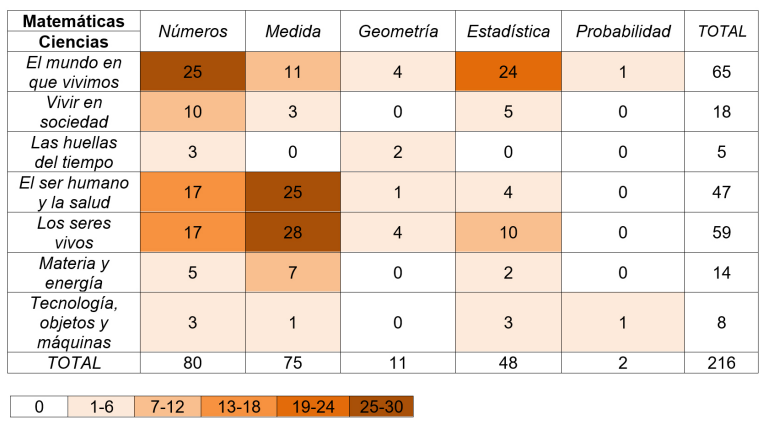

Figura 6. Número de Propuestas Didácticas en las que se trabaja cada contenido
El bloque de ciencias más trabajado por los alumnos es el de "El mundo en que vivimos". Los alumnos relacionan este tema fundamentalmente con el bloque de números y estadística. También destacan en este bloque la geografía y el estudio del universo, relacionados en numerosas propuestas con el sistema métrico decimal. En lo relativo al uso de herramientas TIC, en las Figuras 7 y 8 mostramos la diversidad de recursos utilizados en los trabajos. Cabe destacar el uso de los editores web Wix y Blogger.

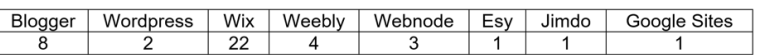

Figura 7. Plataformas de desarrollo web utilizadas en las Propuestas Didácticas analizadas

Los recursos TIC más utilizados, tal y como se detalla en la Figura 8, fueron los programas JClic y HotPotatoes, ya que son recursos que los alumnos conocen por tratarse en otras asignaturas del grado. No obstante, muchos han optado por sustituirlos por otros como Educaplay, ExeLearning o el Constructor Atenex. Cabe destacar también el uso de noticias de periódicos y cuestionarios en la fase de exploración, y de Youtube, presentaciones y libros virtuales (Cuadernia, Emaze, Calameo) en la fase de introducción de conceptos. Los mapas conceptuales (CmapTools, Bubblr) y posters virtuales (Popplet, Glogster) son muy utilizados en la fase de estructuración. Muchos hacen uso de Google Earth y Google Maps para trabajar contenidos de geografía.

\begin{tabular}{|l|l|l|l|}
\hline Recurso & Propuestas & Recurso & Propuestas \\
\hline JClic & $59.5 \%$ & Educaplay, GeoGebra & $16.7 \%$ \\
\hline YouTube & $42.8 \%$ & Prezy & $14.3 \%$ \\
\hline HotPotatoes & $38.1 \%$ & $\begin{array}{l}\text { Pdf, CmapTools, Google Earth } \\
\text { ExeLearning, Google Forms, } \\
\text { Scratch }\end{array}$ & $11.9 \%$ \\
\hline WebQuest & $28.6 \%$ & $\begin{array}{l}\text { Constructor Atenex, Wiki, } \\
\text { Power Point, Wordle, Blogger }\end{array}$ & $9.5 \%$ \\
\hline $\begin{array}{l}\text { Excel, Pizarra } \\
\text { digital }\end{array}$ & $19 \%$ & $\begin{array}{l}\text { Paint, Cuadernia, Emaze, Popplet, Movie- } \\
\text { Maker, Bubble, Pixton, Time-Rime }\end{array}$ & $7.1 \%$ \\
\hline
\end{tabular}

Figura 8. Recursos TIC utilizados en las Propuestas Didácticas analizadas

Respecto al contexto, se observa que la mayoría de los alumnos eligen contextos geográficos cercanos tales como parajes naturales o lugares de la ciudad de Valencia tales como parques zoológicos o botánicos. Esta elección les permite diseñar propuestas que pueden incluir visitas para realizar alguna actividad.

\section{Discusión y conclusiones}

La experiencia realizada durante dos cursos consecutivos en el marco de una asignatura interdisciplinar nos permite identificar aspectos importantes relativos a la formación inicial del profesorado de Educación Primaria en lo referido al ámbito STEM.

A partir del cuestionario en línea, contestado de forma voluntaria por los estudiantes durante el curso 2014-15, deducimos que se valora positivamente el desarrollo de la asignatura, aunque los contenidos impartidos y las actividades realizadas tienen para ellos, en comparación, un interés menor que el resto de aspectos evaluados. Esto puede explicarse por la metodología activa de trabajo de la asignatura, en la que se dedicaron pocas sesiones al tratamiento explícito de contenidos, y muchas al trabajo grupal, dedicadas al diseño de sus propuestas STEM. 
Beltrán Meneu, María José; Hurtado Soler, Desamparados y Ferrando Palomares, Irene (2018) “Formación STEM en el grado de maestro: una experiencia docente". @tic revista d'innovació educativa, 20, 35-42.

Consideramos que esta metodología es adecuada, sin embargo, en vista de los resultados, sería interesante incluir actividades que fueran más allá del diseño, dedicadas al análisis crítico de propuestas e, incluso, de vídeos como los que podemos encontrar en las webs de proyectos tales como Primas ${ }^{2}$ o Lema ${ }^{3}$, de forma que los futuros maestros pudieran no sólo analizar enunciados de tareas, sino también discutir sobre su implementación en el aula. Este tipo de actividades basadas en la observación de vídeos es, según la literatura, enriquecedora en la formación inicial de profesores (Star y Strickland, 2008).

Hemos observado que los futuros maestros tienden a diseñar propuestas STEM dirigidas a alumnos de grado 3 en adelante. Teniendo en cuenta que un maestro STEM debe ser capaz de adaptar una propuesta didáctica a cualquier nivel educativo, así como saber relacionar múltiples contenidos de ciencias y de matemáticas, y tratarlos haciendo uso de las TIC (Moomaw 2013, p. 4), cabe replantearse algunos aspectos de la asignatura. Para promover el diseño de propuestas dirigidas a niveles elementales es necesario, en primer lugar, mostrar a los estudiantes ejemplos claros de propuestas dirigidas a alumnos de corta edad tales como los que pueden encontrarse en repositorios en línea, como, por ejemplo, el que encontramos en la comunidad Scientix ${ }^{4}$. Otro sesgo que hemos detectado en las propuestas diseñadas por los estudiantes, es que tienden a no incluir en sus propuestas contenidos matemáticos de los bloques de geometría y probabilidad. Es posible que esto se explique por el diseño de los estudios de Grados en Maestro/a de Educación Primaria de la Universitat de València (UV). En efecto, cuando inician el diseño de sus trabajos, no han finalizado su formación en didáctica de la geometría y la probabilidad, mientras que sí han superado el resto de asignaturas de didácticas específicas que se asocian con el resto de bloques de contenidos tanto de currículo de matemáticas como de ciencias.

El análisis de las propuestas nos permite inferir que los alumnos las enmarcan en contextos relativamente cercanos a su realidad y que, sin embargo, tienen algunas dificultades a la hora de diseñar actividades de modelización a partir de problemas ubicados en esa misma realidad. Esto se debe a que estas actividades han estado hasta ahora fuera del sistema educativo $\mathrm{y}$, por tanto, los estudiantes son ajenos a ellas ya que no las han implementado como alumnos. En el futuro debemos considerar la posibilidad de trabajar con algo más de detalle algunos aspectos relativos al diseño de tareas de modelización en las sesiones teóricas.

Respecto al uso de las TIC, pese a que, en algunos casos, su uso en el diseño de las propuestas se limita al uso de programas de edición a través de los cuales proponen actividades sobre una plataforma virtual, consideramos que es muy positivo que, para la gran mayoría de estudiantes, nuestra asignatura es de ayuda para conocer y familiarizarse con programas 0 aplicaciones que hasta el momento les eran desconocidas. En este sentido y dado los numerosos cambios que se producen en el entorno de las nuevas tecnologías, es fundamental mostrar la necesidad de actualizarse para ofrecer a sus estudiantes propuestas

\footnotetext{
2 http://primas-project.eu

3 http://www.lema-

project.org/web.lemaproject/web/eu/tout.php

4 http://www.scientix.eu/resources
}

didácticas que se ajusten a sus necesidades educativas. En cualquier caso, durante el desarrollo de las sesiones de clase se observó una mejora de las competencias de los alumnos en el uso de las TIC.

Además de los datos objetivos utilizados en ambos análisis, como responsables de la asignatura durante los últimos años, hemos observado que los futuros maestros, pese a ser críticos con la enseñanza recibida en su infancia, tienden a reproducir en sus propuestas patrones de actividades que aparecen en las propuestas didácticas estándar.

\section{Bibliografía}

Brown, J. (2012) 'The current status of STEM education research', Journal of STEM Education 13(5), 7-11.

Capraro, R. M. y Slough, S. W. (2008) 'Why PBL? Why STEM? Why now? An introduction to STEM project based learning: an integrated science, technology, engineering, and mathematics (STEM) approach', in R.M. Capraro, M.M. Capraro y J. Morgan (eds.), STEM Project-Based Learning: An Integrated Science, Technology, Engineering and Mathematics (STEM) approach, pp. 1-5, Sense Publishers, Rotterdam.

Couso, D. (2013) 'La elaboración de unidades didácticas y competenciales', Alambique: Didáctica de las ciencias experimentales 74, 12-24.

De Pro, A. (2012) '¿Desarrollar competencias matemáticas en las clases de ciencias?', Alambique: Didáctica de las Ciencias Experimentales 70, 54-65.

Díaz Barriga, A. (2011) 'Competencias en educación. Corrientes de pensamiento e implicaciones para el currículo y el trabajo en el aula'. RIES: Revista Iberoamericana de Educación Superior 2(5), 3-24. Disponible

https://ries.universia.net/article/view/61/competencia s-educacion-corrientes-pensamiento-implicacionescurriculo-trabajo-aula. Fecha de consulta, 11/11/2017. https://doi.org/10.22201/iisue.20072872e.2011.5.61

Driver, R. (1988) 'Un enfoque constructivista para el desarrollo del currículo en ciencias', Enseñanza de las Ciencias 6(2), 109-120. Disponible en http://www.raco.cat/index.php/Ensenanza/article/vi ew/51075. Fecha de consulta, 11/11/2017.

English, L. D. y Mousoulides, N. (2011) 'Engineeringbased modelling experiences in the elementary and middle classroom', in M. S. Khine, y I. M. Saleh (eds.), Models and modeling: Cognitive tools for scientific enquiry, pp. 173-194, Springer, Dordrecht.

European Commission/EACEA/Eurydice (2012) Developing Key Competences at School in Europe: Challenges and Opportunities for Policy-2011/12. Eurydice Report. Publications Office of the European Union, Luxembourg.

Jorba, J. y Sanmartí, N. (1996) Enseñar, aprender y evaluar: un proceso de evaluación continua: Propuestas didácticas para las áreas de las ciencias naturales y matemáticas, Ministerio de Educación y Cultura, Centro de Investigación y Documentación Educación (C.I.D.E.). Disponible en https://sede.educacion.gob.es/publiventa/vistaPrevi a. action?cod $=41 \&$ area $=\mathrm{E}$ 
Beltrán Meneu, María José; Hurtado Soler, Desamparados y Ferrando Palomares, Irene (2018) “Formación STEM en el grado de maestro: una experiencia docente". @tic revista d'innovació educativa, 20, 35-42.

Kertil, M. \& Gurel, C. (2016) 'Mathematical modeling: A bridge to STEM education', International Journal of Education in Mathematics, Science and Technology 4(1), 44-55.

Lesh, R. y Zawojewski, J.S. (2007) 'Problem Solving and Modeling', in: Lester, F., (ed.), Second Handbook of Research on Mathematics Teaching and Learning, pp. 763-802, Information Age Publishing, Greenwich.

Lingefjärd, T. (2006) 'Faces of mathematical modeling', ZDM - The International Journal on Mathematics Education 38(2), 96-112.

Niess, M. L. (2005) 'Preparing teachers to teach science and mathematics with technology: Developing a technology pedagogical content knowledge', Teaching and teacher education 21(5), 509-523.

Moomaw, S. (2013) Teaching STEM in the early years: Activities for integrating science, technology, engineering, and mathematics, Redleaf Press.

Reeve, E. (2015) 'STEM thinking!' Technology and Engineering Teacher 74(4), 8-16.

Rinke, C. R., Gladstone-Brown, W., Kinlaw, C. R. y Cappiello, J. (2016) 'Characterizing STEM Teacher Education: Affordances and Constraints of Explicit STEM Preparation for Elementary Teachers', School Science and Mathematics 116(6), 300-309.

Ros, A. C., 2013, 'Hacer unidades didácticas: una tarea fundamental en la planificación de las clases de ciencias', Alambique: Didáctica de las ciencias experimentales 74, 5-11.

Star, J. R. y Strickland, S. K., 2008, 'Learning to observe: Using video to improve preservice mathematics teachers' ability to notice', Journal of mathematics teacher education 11(2), 107-125.

Treffers, A. (1987) Three dimensions: A model of goal and theory description in mathematics instruction The Wiskobas Project, Reidel, Dordrecht, the Netherlands.
Tsupros, N., Kohler, R. y Hallinen, J. (2009) STEM education: A project to identify the missing components. Intermediate Unit 1. Center for STEM Education and Leonard Gelfand Center for Service Learning and Outreach, Carnegie Mellon University, Pennsylvania.

Yasar, S., Baker, D., Kurpius-Robinson, S., Krause, S. y Roberts, C. (2006) 'A valid and reliable survey instrument for measuring K-12 teachers' perceptions and needs on design, engineering, and technology', ASEE Annual Conference and Exposition, Conference Proceedings.

I Cita recomendada de este artículo

Beltrán Meneu, María José; Hurtado Soler, Desamparados y Ferrando Palomares, Irene (2018). “Formación STEM en el grado de maestro: una experiencia docente". @tic revista d'innovació educativa, 20, 35-43. 
Beltrán Meneu, María José; Hurtado Soler, Desamparados y Ferrando Palomares, Irene (2018) “Formación STEM en el grado de maestro: una experiencia docente". @tic revista d'innovació educativa, 20, 35-42.

\section{ANEXO. Evalúa la asignatura "TIC como recurso didáctico en ciencias y matemáticas}

Especifica el nivel de acuerdo o desacuerdo con cada ítem. 0 significa muy en desacuerdo y 5 muy de acuerdo

\begin{tabular}{|c|c|c|c|c|c|c|}
\hline & 0 & 1 & 2 & 3 & 4 & 5 \\
\hline \multicolumn{7}{|l|}{ Estructura y organización de la asignatura } \\
\hline 1. La asignatura es interesante & & & & & & \\
\hline $\begin{array}{l}\text { 2. La forma de evaluación de la asignatura es adecuada } \\
\text { (Examen, propuesta didáctica, coevaluación en la actividad del huerto, exposición, } \\
\text { actividades de modelización) }\end{array}$ & & & & & & \\
\hline $\begin{array}{l}\text { 3. Las actividades son interesantes } \\
\text { (Actividades de modelización, propuesta didáctica, actividad de "juego de roles en el } \\
\text { huerto", Flickr, exposiciones) }\end{array}$ & & & & & & \\
\hline 4. Las clases están bien organizadas y se han logrado los objetivos & & & & & & \\
\hline $\begin{array}{l}\text { 5. Los contenidos teóricos trabajados son interesantes } \\
\text { (Estructura de una propuesta didáctica, actividades de modelización, evaluación, recursos } \\
\text { IIC) }\end{array}$ & & & & & & \\
\hline 6. Se fomenta el trabajo cooperativo & & & & & & \\
\hline \multicolumn{7}{|l|}{ Trabajo final: Propuesta Didáctica } \\
\hline \multicolumn{7}{|l|}{$\begin{array}{l}\text { 7. La estructura de la propuesta didáctica es adecuada } \\
\text { (actividades de exploración, de introducción de conceptos, de estructuración y de } \\
\text { aplicación) }\end{array}$} \\
\hline 8. Me ha resultado difícil elegir el contexto para la propuesta didáctica & & & & & & \\
\hline \multicolumn{7}{|l|}{$\begin{array}{l}\text { 9. Es interesante fijar un contenido de ciencias y uno de matemáticas común a } \\
\text { todas las propuestas }\end{array}$} \\
\hline \multicolumn{7}{|l|}{ 10. Es difícil relacionar los contenidos fijados con el contexto } \\
\hline \multicolumn{7}{|l|}{ 11. El tiempo de trabajo es adecuado } \\
\hline \multicolumn{7}{|l|}{ 12. Es difícil trabajar en grupo } \\
\hline \multicolumn{7}{|l|}{ 13. Las fechas de exposición y entrega son adecuadas } \\
\hline \multicolumn{7}{|l|}{ 14. Las exposiciones previas son útiles } \\
\hline \multicolumn{7}{|l|}{ 15. Los ítems de la hoja de evaluación de la propuesta son adecuados } \\
\hline \multicolumn{7}{|l|}{ Actividad de modelización } \\
\hline \multicolumn{7}{|l|}{$\begin{array}{l}\text { 16. Las actividades de modelización son interesantes para llevarse a cabo con } \\
\text { alumnos de primaria }\end{array}$} \\
\hline \multicolumn{7}{|l|}{$\begin{array}{l}\text { 17. Es difícil diseñar actividades de modelización relacionadas con el contexto de la } \\
\text { propuesta didáctica }\end{array}$} \\
\hline \multicolumn{7}{|l|}{$\begin{array}{l}\text { 18. Las actividades de modelización han contribuido al aprendizaje de las } \\
\text { matemáticas }\end{array}$} \\
\hline \multicolumn{7}{|l|}{ 19. Es difícil resolver actividades de modelización } \\
\hline \multicolumn{7}{|l|}{ Actividad de "Juego de roles en el Huerto" } \\
\hline \multicolumn{7}{|l|}{$\begin{array}{l}\text { 20. La actividad realizada en el huerto es interesante para llevarse a cabo con } \\
\text { alumnos de primaria }\end{array}$} \\
\hline \multicolumn{7}{|l|}{$\begin{array}{l}\text { 21. Es difícil diseñar actividades en el huerto relacionadas con el contexto de la } \\
\text { propuesta didáctica }\end{array}$} \\
\hline \multicolumn{7}{|l|}{ 22. La actividad de "Juego de roles en el huerto" ha contribuido a vuestro aprendizaje } \\
\hline \multicolumn{7}{|l|}{ Uso de herramientas "TIC" } \\
\hline \multicolumn{7}{|l|}{ 23. Se han descubierto nuevas herramientas "TIC" para crear actividades } \\
\hline \multicolumn{7}{|l|}{ 24. Se han utilizado herramientas TIC distintas a las usadas en cursos anteriores } \\
\hline \multicolumn{7}{|l|}{ 25. Se han encontrado dificultades técnicas con el uso de las TIC } \\
\hline \multicolumn{7}{|l|}{ 26. Se ha logrado superar las dificultades técnicas con el uso de las TIC } \\
\hline 27. Se han mejorado la competencias en el uso de las TIC & & & & & & \\
\hline
\end{tabular}

